\title{
FLYING IN NORTH AMERICA
}

\author{
Differences
}

\author{
Jaromír Procházka \\ Department of Air Transport, \\ Department of Air Transport, Faculty of Transportation Sciences, Czech Technical University \\ Horská 3, Praha 2, 128 03, Czech Republic \\ e-mail: xjprochazka@fd.cvut.cz
}

\begin{abstract}
Flying all over the world is very challenging and demanding. Although ICAO is covering every specific detail by rules, there are some personal experiences which cannot be substituted. Some of them are point out in the article.
\end{abstract}

Key words: Uncontrolled Airport; FAA; IFR; Differences in North America;

\section{INTRODUCTION}

Pilots operating mostly in Europe region are familiar with local procedures and some specific type of operations very well. Nevertheless operations in different continent may bring a new opportunity how to deal with unexpected aspects. It is worth to be prepared and following pages may be taken as a quick review of main differences during operating in North America. There are highlighted three sections which could be taken in account. The first one is weather because without any doubt it is a one of main factors for safe operations. The second one are procedures used in uncontrolled airports. There are not much uncontrolled airports in Europe which are operated by aircraft as Boeing 737 or A320 and be aware of this author describing in details the procedures which must be followed. The last section is only short description pilot control of aerodrome lighting.

\section{WEATHER}

\section{A. General Condition}

Weather systems generally move from the west or southwest into the eastern regions. During the winter season storms passing through the Maritimes, Newfoundland and eastern United States frequently grow in intensity on reaching coastal areas. These briskly moving weather systems can cause rapid changes in the weather patterns.

\section{B. Local Phenomena}

The south-western Ontario region is surrounded by water, which has a considerable effect on the weather in this area. During the winter months heavy snow accumulations can occur with lake effect snows from the Great Lakes. In the summer months thunderstorm activity is expected and can affect departure and arrivals and can cause deviations off route. Thunderstorms are often imbedded or hidden in haze at lower altitudes.

\section{Jetstreams}

Jetstreams and jetstreaks in North America play a big role all year round generating strong head and tailwinds as well as all levels of turbulence. Routes across the southern prairies are subject to thunderstorm activity. Historically, heavier activity is normally along the US/Canada border. Routes across the Rockies can be quite turbulent especially if there is a strong southwest flow aloft. This can cause mountain wave activity that can extend as far as central Canada/USA as a result of resonance activity. The descent into the West Coast stations can also be quite turbulent.

\section{Seasonal effects}

During fall and winter, tornadoes are confined to gulfbordering states, in spring the activity shifts northward to Kansas and Missouri. By May and June tornado activity extends from Oklahoma to occasional activity in southern Canada. Tornados have occurred as far north as Edmonton, Alberta along a line to the east through Lake Manitoba and Winnipeg. Intensity diminishes during summer and recedes southward again as fall approaches. Tornadoes occur at all hours, in all central states, and as late as November over the central plains. The area lying inland from the Sierra Nevada and the Cascades has a winter or rainy season similar to Vancouver from November to March and a dry season from May to November.

\section{E. Whiteout}

Whiteout is an atmospheric optical phenomenon in which the observer appears to be engulfed in a uniformly white glow. Shadows, the horizon nor clouds are discernable. Sense of depth and orientation are lost and only very dark nearby objects can be seen. Whiteout occurs over an unbroken snow cover and beneath a uniformly overcast sky when, with the aid of the snowblink effect, the light from the sky is about equal to

Identify applicable sponsor/s here. (sponsors) 
that from the snow surface. Blowing snow may also be a contributing factor.

Whenever a pilot encounters the whiteout conditions described above, the pilot should immediately climb if at low level. The flight should not proceed unless the pilot can safely traverse the whiteout area on instruments.

In addition, the following phenomena are known to cause whiteout and should be avoided if at all possible:

a) WATER-FOG WHITEOUT RESULTING FROM THIN CLOUDS OF SUPER-COOLED WATER DROPLETS IN CONTACT WITH THE COLD SNOW SURFACE. DEPENDING ON THE SIZE AND DISTRIBUTION OF THE WATER DROPLETS, VISIBILITY MAY BE MINIMAL OR NIL IN SUCH CONDITIONS.

b) BLOWING SNOW WHITEOUT RESULTING FROM FINE SNOW BEING PLUCKED FROM THE SURFACE BY WINDS OF 20 KT OR MORE. SUNLIGHT IS REFLECTED AND DIFFUSED RESULTING IN A NIL VISIBILITY WHITEOUT CONDITION.

c) PRECIPITATION WHITEOUT RESULTING FROM SMALL WIND-DRIVEN SNOW CRYSTALS FALLING FROM LOW CLOUDS ABOVE WHICH THE SUN IS SHINING. LIGHT REFLECTION COMPLICATED BY SPECTRAL REFLECTION FROM THE SNOW FLAKES AND OBSTRUCTION OF LAND MARKS BY FALLING SNOW CAN REDUCE VISIBILITY AND DEPTH PERCEPTION TO NIL IN SUCH CONDITION.

\section{UNCONTROLLED AIRPORT PROCEDURES}

Each pilot has own experience with uncontrolled airport mostly from his private pilot license training. Nevertheless is quite different to operate such aerodromes in commercial transport aviation with medium or heavy jet airplane. There are some procedures that must be kept in mind. Here is presented short draft how to do that.

\section{A. Arrivals}

Pilot shall establish communications with the air to ground facility when directed to do so. The pilot intending to conduct an approach to an uncontrolled airport shall report:

a) Five minutes prior to commencing the approach, stating the estimated landing time

b) When commencing a circling maneuver

c) As soon as practicable after initiating a missed approach procedure

d) The aircrafts position when passing the fix outbound when intending to do a procedure turn or when aircraft first intercepts the final approach course, when passing the FAF (or 3 minutes before landing if no FAF exists), and on final approach

IFR clearance must be cancelled either in the air or on ground via ATC or by phone. Frequency 126.7 should be monitored while operating in uncontrolled airspace and broadcast their intentions prior to changing altitudes or commencing the approach.

\section{B. Departures}

Where a pilot in command intends to take off from an uncontrolled aerodrome, the pilot shall:

a) Obtain an ATC clearance if in controlled airspace

b) Report on the appropriate frequency their departure procedure and intentions before moving on the runway or before aligning the aircraft on the take off path

c) Ascertain by radio on the appropriate frequency and by visual observation that no other aircraft or vehicle is likely to come into conflict with the aircraft during take off

d) The pilot shall maintain a listening watch during takeoff from an uncontrolled aerodrome

e) After takeoff from an uncontrolled airport, until the aircraft is beyond the distance or above the altitude associated with the frequency

As soon as possible after reaching the distance or altitude, the pilot in command shall communicate with the appropriate ATC unit of ground station on the appropriate en route frequency. Where IFR departures are required to contact an IFR control unit or ground station after takeoff, it is recommended that if the aircraft is equipped with two radios the 126,7 frequency should also be monitored. If the airport is located in uncontrolled airspace, these procedures shall be followed except that an ATC clearance is not required. In addition to maintaining a listening watch, it is recommended that that the pilot communicates with the appropriate ATC unit or other ground station on the appropriate en route frequency.

It is recommended that pilots inform ATC if a flight will not commence within 60 minutes of the proposed departure time stipulated in an IFR flight plan. Failure to do so will result in activating the Search and Rescue (SAR) process.

At an uncontrolled aerodrome, the initial IFR clearance may contain a time or an event based departure restriction or clearance cancellation.

\section{Clearance}

When cleared for "an approach" to an aerodrome, the pilot has the option of conducting any published approach to that aerodrome. ATC must be advised of the intended instrument approach procedure being conducted. No deviations should be made from the stated instrument approach procedure without consent form ATC. Such an act cause dangerous conflict with another aircraft or a vehicle on the runway. Descent to the appropriate IFR altitude (minimum en route altitude, transition altitude, safe altitude $100 \mathrm{NM}$, or minimum sector altitude is at the pilots discretion. But descent to the safe altitude $100 \mathrm{NM}$ or 
the MSA may take the aircraft out of controlled airspace ATC provides IFR separation only within controlled airspace.

Note: Approach clearance is for a published IAP. Visual approaches are authorized at uncontrolled aerodromes. A contact approach can be authorized at the pilots request.

\section{Frequencies}

Whether or not the aircraft is operating under ATC control, it remains the pilots responsibility to notify the ground station at the destination aerodrome (or to broadcast, where no ground station exists) and report in accordance with current aviation regulations.

\section{E. Communications}

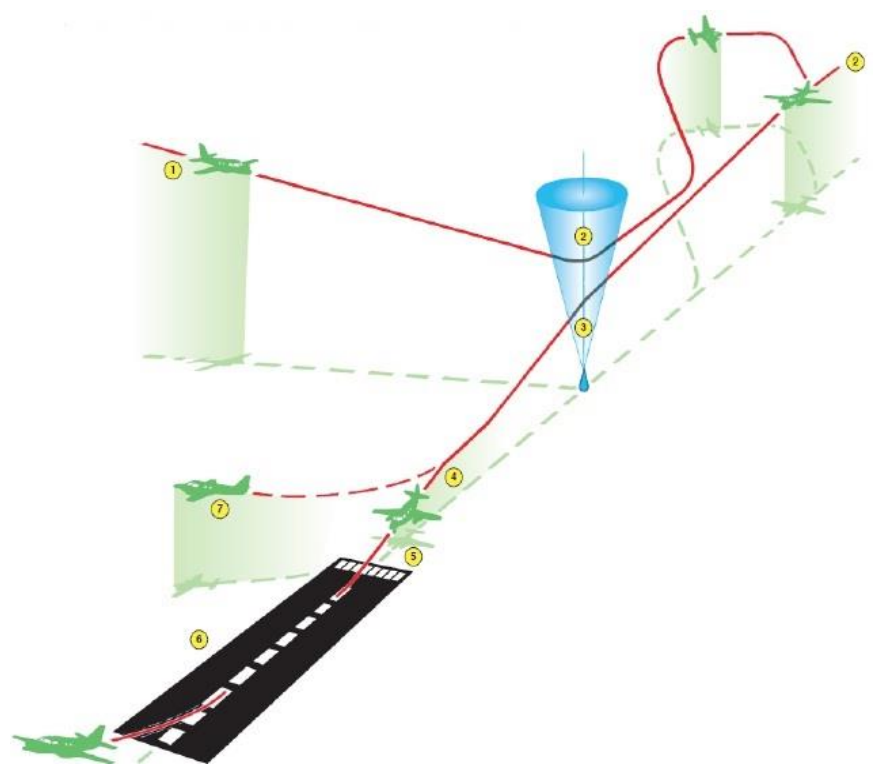

1 - Report 5 min prior to the estimated time of commencing the approach include the intended approach procedure and estimated time of landing.

2 - Report when passing the fix with the intention of conducting a procedure turn. If no turn procedure is intended report intercepting the final approach track.

3 - Report when passing the final approach fix inbound of where no final approach fix exists report $3 \mathrm{~min}$ before the estimated time of landing.

4 - Ensure the runway is clear prior to landing.

5 - Advise intentions upon commencing a circling procedure and when turning onto final.

6 - Report when clear of all runways after landing and ensure the flight plan is closed.

7 - In the event of a missed approach, report intentions as soon as practicable.

\section{F. Uncontrolled airspace - en route}

Monitor $126.7 \mathrm{MHz}$ whenever practical. Broadcast intentions before any changes in altitude and before commencing an approach. On arrival, broadcast intentions on 126.7 MHz within 15 min of the estimated time of arrival and prior do descent. Then report on the appropriate aerodrome frequency $5 \mathrm{~min}$ commencing the approach.

\section{G. Special departure procedures}

On the appropriate frequency: (Pilots departing IFR should also broadcast on $126.7 \mathrm{MHz}$ )

a) Report departure procedure and intentions befoere moving onto the runway.

b) Ascertain that no conflict will occur with vehicles or other aircraft during takeoff (confirm by radio and by visual scan).

c) Continue the listening watch from takeoff until beyond the distance and/or above the altitude associated with the frequency.

d) Contact ATC on the appropriate frequency as soon as practicable after reaching the distance and altitude associated with the aerodrome frequency.

An IFR clearance does not confer special priority. In visual meteorological conditions, you must safely fit in with established VFR traffic.

\section{AIRCRAFT RADIO CONTROL OF AERODROME LIGHTING}

Uncontrolled airports sometimes use ARCAL (aircraft radio control of aerodrome lighting), this requires pilots interaction to operate.

\section{A. Type J}

To operate all aerodrome lighting for duration of approximately 15 minutes key microphone 5 times within 5 seconds. The timing cycle may be restarted at anytime by repeating the keying sequence. Some systems will indicate when the duration period is over by flashing once then remaining on for a further 2 minutes before extinguishing. Some systems do not give you this warning. The control system may operate $\mathrm{H} 24$ or between sunset and sunrise.

\section{B. Type $K$}

To operate all aerodrome lighting for duration of approximately 15 minutes, key microphone 7 times initially. This will ensure all lights are on maximum intensity. The intensity may be adjusted up or down to any one of three settings by keying the microphone 7,5 , or 3 , times within 5 seconds for high, medium, or low intensity settings respectively. The timing cycle may be restarted at any time by repeating the initial keying sequence. Where runway Identification Lights are available, keying the 
microphone 3 times on the appropriate frequency will turn them off.

\section{REFERENCES}

[5] www.icao.in

[1] www.faa.gov

[2] www.swgops.ca

[3] www.iata.org

[4] www.caa.cz 